\title{
A INCONTINÊNCIA URINÁRIA EM IDOSOS E FATORES ASSOCIADOS: UMA REVISÃO SISTEMÁTICA
}

\section{ARTIGO DE REVISÃO}

JESUS, Giselle Helena Monteiro de ${ }^{1}$

MELO, Amanda Cristina Lobo de ${ }^{2}$

COELHO, Cybelle Silva do Couto $^{3}$

GALÚCIO, Vanessa Costa Alves ${ }^{4}$

JESUS, Giselle Helena Monteiro de. Et al. A Incontinência Urinária em idosos e fatores associados: uma revisão sistemática. Revista Científica Multidisciplinar Núcleo do Conhecimento. Ano 05, Ed. 07, Vol. 04, pp. 05-16. Julho de 2020. ISSN: 2448-0959, Link de acesso: https://www.nucleodoconhecimento.com.br/saude/incontinencia-urinaria

\section{RESUMO}

Introdução: $O$ envelhecimento humano traz inúmeros desafios para o cuidado decorrente de patologias crônicas existentes, incluindo a Incontinência Urinária (IU) atingindo principalmente mulheres idosas. No entanto a IU interfere diretamente na qualidade de vida de idosos no Brasil e em outros países. De acordo com a

${ }^{1}$ Graduação em Biomedicina.

2 Graduação em Biomedicina.

3 Pós-graduação em Fisioterapia Traumato Ortopédica e Desportiva; Graduação em Fisioterapia.

${ }^{4}$ Orientadora. Doutorado em Biotecnologia. Mestrado em Biotecnologia e Recursos Naturais da Amazônia. Especialização em Análise Clínica e Microbiologia. Especialização em Gestão da Segurança de Alimentos. Especialização em Educação Ambiental. Graduação em Biomedicina. 
International Continence Society (ICS), a IU é caracterizada pela perda involuntária da urina que são chamados: incontinência urinária de urgência, de esforço e mista. Esta perda também pode estar relacionada com a fraqueza do assoalho pélvico, com a contração involuntária da musculatura pélvica e até mesmo a perda da sensibilidade. Objetivo: Analisar, por meio de revisão sistemática da literatura, as causas da Incontinência Urinária em idosos, com a perspectiva de identificar os principais fatores de risco associados, bem como identificar possíveis processos de reversão do quadro. Materiais e métodos: Foi realizada uma revisão sistemática da literatura por meio de pesquisa de artigos publicados no período de 2010 a 2019, utilizando as bases de dados SCIELO, LILACS, BIREME e PUBMED. Foram analisados 30 artigos em inglês e português, selecionados de acordo com os descritores: incontinência urinária, envelhecimento, saúde do idoso e fatores de risco, onde obteve-se 14 artigos elegíveis. Foram excluídos artigos que não atenderam integralmente o objetivo e artigos duplicados. Discussão e Resultados: Foram encontrados inúmeros fatores que podem levar ao quadro de incontinência urinária, e dentre eles os de risco associado à com morbidades. A literatura aponta que na população idosa um dos fatores mais comuns são o enfraquecimento do assoalho pélvico e perda de mobilidade articular e que, além destes, existem doenças e condições que podem acarretar esse processo patológico. Com base nisto, o tratamento vem se tornando cada vez mais claro e multidisciplinar, iniciando desde o momento de diagnóstico, através de exames laboratoriais de rotina e específicos, até o tratamento medicamentoso, através da antibioticoterapia e terapêutico, com a fisioterapia pélvica. Conclusão: O risco de ocorrência da incontinência urinária no decorrer da vida esta associado a fatores diversos, afetando diretamente a qualidade de vida, principalmente da população idosa.

Palavras-Chave: Incontinência urinaria, envelhecimento, saúde do idoso, fatores de risco.

\section{INTRODUÇÃO}

O envelhecimento humano traz consigo inúmeros desafios para o cuidado decorrente de patologias crônicas existentes, podendo incluir a Incontinência Urinária (IU) como 
um dos casos recorrentes, principalmente em mulheres idosas com faixa etária acima de 60 anos. $^{1}$

A incontinência urinária (IU) é estabelecida como uma perda involuntária de urina, além de ser um distúrbio que acomete mais comumente, o gênero feminino e de população mais idosa, de acordo com o Ministério da Saúde. ${ }^{2}$ Esta patologia pode gerar problemas para um envelhecer saudável e com qualidade, interferindo diretamente na qualidade de vida de idosos tanto no Brasil, quanto em outros países. ${ }^{3}$

Entretanto, é importante salientar que a prevalência da IU aumenta com o envelhecimento tanto de mulheres e homens, devido ao envelhecimento fisiológico normal em ambos os gêneros. ${ }^{4}$ No estudo de Cavalcante et al, é citado que grupos de idosos obtinham prevalência da IU em $55 \%$ dos homens e $69 \%$ das mulheres. ${ }^{1}$

De acordo com a International Continence Society (ICS), a IU é caracterizada pela perda involuntária de um volume de urina, sendo descrita pela primeira vez durante o ano de 1616, onde o distúrbio foi estudado por MV Goldberg. ${ }^{5}$ Esta perda também pode estar relacionada com o enfraquecimento do assoalho pélvico, com a contração involuntária da musculatura pélvica ou até mesmo a perda ou alteração da sensibilidade nesta região, e também pode ser desencadeada através de processos patológicos, tais como a hipertensão arterial, diabetes mellitus, números de partos normais, infecção do trato urinário recorrente, uso de medicamentos diuréticos, vasodilatadores, anti-histamínicos, sedativos, tranquilizantes e narcóticos. ${ }^{6}$

Com base nisto, o tratamento vem se tornando cada vez mais claro e multidisciplinar, iniciando desde o momento de diagnóstico, através de exames laboratoriais de rotina e específicos, até o tratamento medicamentoso, através da antibioticoterapia e terapêutico, com a fisioterapia pélvica além de haver diferentes tipos de manifestações clínicas. ${ }^{7}$

Para diferenciar essas manifestações, a incontinência urinária possui 3 classificações: incontinência urinária de urgência, de esforço e mista. ${ }^{8} \mathrm{Na}$ forma de urgência, a perda de urina é acompanhada ou imediatamente antecedida por uma vontade súbita ou 
urgência miccional, o que é mais frequente na mulher idosa, tendo em vista o envelhecimento fisiológico; na forma de esforço, caracteriza a perda de urina relacionada com o esforço, sem sensação de vontade de urinar ou de "bexiga cheia", que pode ocorrer predominantemente na mulher jovem e em idade adulta, mais frequentemente após muitos partos; e na forma mista, há uma caracterização de ambas as formas, independente de idade..$^{9}$

Desse modo, avaliar e conhecer os fatores de risco, bem como as formas de tratamento para a incontinência urinária são importantes para o desenvolvimento dos profissionais e estimular os benefícios para a qualidade de vida da população idosa. Portanto, o objetivo desse estudo é analisar, por meio de revisão sistemática da literatura, as causas da Incontinência Urinária em idosas, com a perspectiva de identificar os principais fatores de risco associados a esta patologia, bem como identificar de que forma a medicina trabalha os processos de reversão do quadro.

\section{MATERIAIS E MÉTODOS}

O presente trabalho trata-se de uma revisão sistemática da literatura por meio de pesquisa de artigos publicados no período de 2010 a 2019, utilizando as bases de dados SCIELO, LILACS, BIREME e PUBMED. Foram encontrados 60 artigos elegíveis de análise, e posteriormente analisados 30 artigos em inglês e português, sendo incluídos artigos que tratassem do tema da incontinência urinária no recorte etário de 0 a 05 anos de idade, publicados com o tempo superior a 10 anos, que consideravam aspectos relacionados à IU em mulheres idosas e que apresentavam os descritores: incontinência urinária, envelhecimento, saúde do idoso e fatores de risco (quadro 1), onde obteve-se 14 artigos incluídos. Foram excluídos 10 artigos que não se relacionavam com os critérios de inclusão, que não obtinham aspectos relacionados à IU, como a recorrência de incontinência urinária, forma clínica, causas ou aspectos relacionados a IU e 6 artigos duplicados.

Quadro 1: Equações de buscas em bases de dados. 


\begin{tabular}{|l|l|}
\hline PUBMED & Old woman and urinary incontinence \\
\hline BIREME & $\begin{array}{l}\text { Incontinência urinária fatores sociodemográficos e } \\
\text { comportamentais em idosas. }\end{array}$ \\
\hline LILACS & Incontinência urinária e envelhecimento \\
\hline SCIELO & Incontinência urinaria ou saúde do idoso \\
\hline
\end{tabular}

\section{RESULTADOS}

$\mathrm{Na}$ base inicial do levantamento sistemático, encontrou-se um total de 2154 referências sendo: 491 artigos no PUBMED, 1630 artigos no SCIELO, 9 artigos encontrados na LILACS e 24 artigos na BIREME. Após a leitura dos artigos, foi feita uma triagem e, dela, selecionou-se 60 artigos elegíveis em língua inglesa e língua portuguesa e sendo incluído somente 14 artigos. As características metodológicas de cada artigo podem ser observadas no quadro 2 .

Quadro 02: Características das fontes bibliográficas, tipos de fontes, estudo e local da pesquisa.

\begin{tabular}{|c|c|c|c|}
\hline $\begin{array}{l}\text { ESTUDOS } \\
\text { (AUTORIA E ANO) }\end{array}$ & $\begin{array}{l}\text { TIPO } \quad \text { DE } \\
\text { FONTE }\end{array}$ & TIPO DE ESTUDO & LOCAL \\
\hline $\begin{array}{l}\text { Carvalho et } \\
\text { al. (2014) }\end{array}$ & SCIELO & Transversal & Pelotas (RS) \\
\hline Bolina et al. (2013) & SCIELO & $\begin{array}{l}\text { Observacional } \quad \text { e } \\
\text { transversal }\end{array}$ & Uberaba (MG) \\
\hline $\begin{array}{l}\text { Cavalcante et al. } \\
(2014)\end{array}$ & SCIELO & Transversal & Petrolina (PE) \\
\hline $\begin{array}{l}\text { Carneiro et al. } \\
(2017)\end{array}$ & LILACS & $\begin{array}{l}\text { Transversal de base } \\
\text { populacional }\end{array}$ & $\begin{array}{l}\text { Minas Gerais } \\
(M G)\end{array}$ \\
\hline $\begin{array}{l}\text { Junqueira } \quad \& \\
\text { Santos (2018) }\end{array}$ & SCIELO & $\begin{array}{l}\text { Observacional, } \\
\text { transversal, analítico e } \\
\text { descritivo }\end{array}$ & $\begin{array}{l}\text { Hospital São } \\
\text { Paulo (HU-SP) }\end{array}$ \\
\hline
\end{tabular}




\begin{tabular}{|c|c|c|c|}
\hline $\begin{array}{l}\text { Kessler et al. } \\
(2018)\end{array}$ & PUMED & Estudo transversal & Bagé (RS) \\
\hline Melo et al. (2017) & SCIELO & Coorte, Transversal & $\begin{array}{l}\text { Belo Horizonte } \\
\text { (MG) }\end{array}$ \\
\hline $\begin{array}{l}\text { Marques et al. } \\
(2015)\end{array}$ & SCIELO & Transversal & $\begin{array}{l}\text { Florianópolis, } \\
\text { (SC) }\end{array}$ \\
\hline $\begin{array}{l}\text { Menezes et al. } \\
(2016)\end{array}$ & PUBMED & $\begin{array}{l}\text { Analítico de corte } \\
\text { transversal }\end{array}$ & $\begin{array}{l}\text { Florianópolis } \\
\text { (SC) }\end{array}$ \\
\hline $\begin{array}{l}\text { Murukesu, Singh, } \\
\text { Shahar (2019) }\end{array}$ & BIREME & Longitudinal & Malásia \\
\hline $\begin{array}{l}\text { Mourão et al. } \\
(2017)\end{array}$ & LILACS & $\begin{array}{l}\text { Exploratório, descritivo, } \\
\text { transversal }\end{array}$ & Teresina $(\mathrm{PI})$ \\
\hline $\begin{array}{l}\text { Santos, Santo } \\
(2010)\end{array}$ & BIREME & $\begin{array}{l}\text { Exploratório, descritivo, } \\
\text { transversal }\end{array}$ & $\begin{array}{l}\text { Minas Gerais } \\
(\mathrm{MG})\end{array}$ \\
\hline Silva et al. (2012) & SCIELO & Transversal quantitativa & $\begin{array}{l}\text { São Paulo } \\
\text { (SP) }\end{array}$ \\
\hline $\begin{array}{l}\text { Virtuoso et al. } \\
(2015)\end{array}$ & PUBMED & Analítico transversal & Florianópolis \\
\hline
\end{tabular}

No quadro 3, pode-se observar uma organização de acordo com os critérios metodológicos das pesquisas estudadas, levando em consideração: autoria e ano, os objetivos do estudo, as medidas de avaliação, as frequências da IU em porcentagem versus o tamanho da amostra, bem como os resultados.

Quadro 03: Organização bibliográfica de acordo com autoria, ano, objetivo, frequência de IU \%, e resultados encontrados.

\begin{tabular}{|l|l|l|l|}
\hline $\begin{array}{l}\text { AUTOR E } \\
\text { ANO }\end{array}$ & OBJETIVO & $\begin{array}{l}\text { FREQUÊNCIA } \\
\text { IU \% }\end{array}$ & RESULTADOS \\
\hline
\end{tabular}




\begin{tabular}{|c|c|c|c|}
\hline $\begin{array}{l}\text { Carvalho et } \\
\text { al. (2014) }\end{array}$ & $\begin{array}{l}\text { Identificar a } \\
\text { prevalência de } \\
\text { incontinência } \\
\text { urinária (IU) e } \\
\text { fatores associados } \\
\text { em idosas da } \\
\text { comunidade. }\end{array}$ & $40.91 \%$ & $\begin{array}{l}\text { - Números de gestações são } \\
\text { apontados como causa de } \\
\text { IU. } \\
\text { - Poucas idosas relatam } \\
\text { sobre impacto na qualidade } \\
\text { de vida, sendo o importante } \\
\text { um diagnóstico precoce } \\
\text { para um possível tratamento } \\
\text { para evitar possíveis } \\
\text { complicações e promover } \\
\text { qualidade de vida. }\end{array}$ \\
\hline $\begin{array}{l}\text { Bolina et } \\
\text { al. (2013) }\end{array}$ & $\begin{array}{l}\text { Verificar os fatores } \\
\text { sociodemográficos } \\
\text { e as morbidades } \\
\text { auto referidas } \\
\text { associadas a IU. }\end{array}$ & $52,6 \%$ & $\begin{array}{l}\text { Nesse estudo foi possível } \\
\text { concluir que a infecção do } \\
\text { trato urinário é uma } \\
\text { condição frequente em } \\
\text { idosos com IU com critérios } \\
\text { de fragilidade. }\end{array}$ \\
\hline $\begin{array}{l}\text { Cavalcante } \\
\text { et al. } \\
(2014)\end{array}$ & $\begin{array}{l}\text { Identificar a } \\
\text { prevalência da } \\
\text { incontinência } \\
\text { urinária e os } \\
\text { fatores associados } \\
\text { para cada sexo } \\
\text { entre idosos não } \\
\text { institucionalizados }\end{array}$ & $31 \%$ & $\begin{array}{l}\text { A amostra do estudo foi de } \\
686 \text { indivíduos, dos quais } \\
445(64,9 \%) \text { eram do sexo } \\
\text { feminino. } \\
\text { Houve alta prevalência de } \\
\text { incontinência urinária em } \\
\text { idosos de ambos os sexos. } \\
\text { Fatores relativos a } \\
\text { condições de saúde } \\
\text { estiveram associados à } \\
\text { ocorrência em cada sexo, } \\
\text { mostrando a necessidade }\end{array}$ \\
\hline
\end{tabular}




\begin{tabular}{|c|c|c|c|}
\hline & & & $\begin{array}{l}\text { de uma assistência que } \\
\text { reconheça tais sinais e } \\
\text { sintomas. }\end{array}$ \\
\hline $\begin{array}{l}\text { Carneiro et } \\
\text { al. (2017) }\end{array}$ & $\begin{array}{lr}\text { Verificar } & \text { a } \\
\text { prevalência da } & \text { de } \\
\text { queixa } & \\
\text { Incontinência } & \\
\text { Urinária (IU) e os } \\
\text { fatores associados } \\
\text { em idosas no } \\
\text { município de } \\
\text { Petrolina/PE. }\end{array}$ & $47,1 \%$ & $\begin{array}{l}\text { No estudo houve elevada } \\
\text { prevalência de IU em idosas } \\
(47,1 \%) \text { cujo perfil } \\
\text { majoritário foi de mulheres } \\
\text { que tinha idade superior a } \\
65 \text { anos, viviam sem } \\
\text { parceiro } \\
\text { (separada/viúva/divorciada), } \\
\text { tinham baixa escolaridade, } \\
\text { baixa renda, aposentadoria } \\
\text { de até um salário mínimo, } \\
\text { mas exercendo a função de } \\
\text { chefe de família. }\end{array}$ \\
\hline $\begin{array}{l}\text { Junqueira } \\
\& \quad \text { Santos } \\
(2018)\end{array}$ & $\begin{array}{l}\text { Verificar a } \\
\text { prevalência da IU, } \\
\text { e sua relação com } \\
\text { indicadores de } \\
\text { saúde físico } \\
\text { mental em idosas } \\
\text { de Bagé (RS) }\end{array}$ & $60 \%$ & $\begin{array}{l}\text { Os resultados deste estudo } \\
\text { mostram a } \\
\text { incontinência urinária como } \\
\text { uma condição frequente } \\
\text { entre os idosos, alcançando } \\
\text { um a cada cinco indivíduos. } \\
\text { Além disso, sua prevalência } \\
\text { é maior }\end{array}$ \\
\hline $\begin{array}{l}\text { Kessler et } \\
\text { al. (2018) }\end{array}$ & $\begin{array}{l}\text { Analisar a } \\
\text { prevalência de } \\
\text { incontinência } \\
\text { urinária e seus } \\
\text { fatores associados }\end{array}$ & $22,9 \%$ & $\begin{array}{l}\text { Neste estudo, realizado com } \\
319 \text { pacientes hospitalares, } \\
\text { concluiu-se que a } \\
\text { prevalência geral de IU foi } \\
\text { de } 22,9 \%, 28 \% \text { em mulheres } \\
\text { e } 16,1 \% \text { em homens. }\end{array}$ \\
\hline
\end{tabular}


em pacientes

hospitalares.
Melo et al. (2017)
Determinar
a $97 \%$
prevalência e os
fatores
associados à
incontinência
urinária na
população idosa
de Florianópolis,
Estado de Santa
Catarina.

\begin{tabular}{|c|c|c|c|}
\hline $\begin{array}{l}\text { Marques et } \\
\text { al. (2015) }\end{array}$ & $\begin{array}{l}\text { Avaliar aspectos } \\
\text { epidemiológicos } \\
\text { da infecção do } \\
\text { trato urinário em } \\
\text { pacientes idosos } \\
\text { com incontinência } \\
\text { urinária, } \\
\text { residentes em } \\
\text { instituições de } \\
\text { longa } \\
\text { permanência, de } \\
\text { Belo Horizonte. }\end{array}$ & $3 \%$ & $\begin{array}{l}\text { As variáveis que se } \\
\text { associaram a ocorrência de } \\
\text { ITU foram Bacteriúria e } \\
\text { Instituição. No entanto, ao } \\
\text { analisar a variável "Uso de } \\
\text { Fraldas" foi possível } \\
\text { perceber aumento do risco } \\
\text { de ocorrência de ITU nesses } \\
\text { pacientes. }\end{array}$ \\
\hline $\begin{array}{l}\text { Menezes } \\
\text { et al. } \\
(2016)\end{array}$ & $\begin{array}{l}\text { Determinar a } \\
\text { precisão } \\
\text { diagnóstica e o } \\
\text { ponto de corte das } \\
\text { variáveis } \\
\text { índice de } \\
\text { conicidade, razão }\end{array}$ & $32,2 \%$ & $\begin{array}{l}\text { - A prevalência de IU foi de } \\
32,2 \% \text {. } \\
\text { - Os sintomas de perda } \\
\text { urinária durante os esforços } \\
\text { foram relatados por } 28,3 \% \text {, } \\
\text { enquanto a perda urinária }\end{array}$ \\
\hline
\end{tabular}




\begin{tabular}{|c|c|c|c|}
\hline & $\begin{array}{l}\text { cintura / estatura e } \\
\text { percentual de } \\
\text { gordura para } \\
\text { detectar } \\
\text { incontinência } \\
\text { urinária } \quad \text { em } \\
\text { mulheres idosas } \\
\text { ativas }\end{array}$ & & $\begin{array}{l}\text { associada ao desejo de } \\
\text { urinar foi de } 11,2 \% \text {. } \\
\text { - Para } 7,2 \% \text { da amostra, } \\
\text { ambos os sintomas estavam } \\
\text { presentes. }\end{array}$ \\
\hline $\begin{array}{l}\text { Murukesu, } \\
\text { Singh, } \\
\text { Shahar } \\
(2019)\end{array}$ & $\begin{array}{l}\text { Investigar a } \\
\text { prevalência, } \\
\text { fatores de risco } \\
\text { para incontinência } \\
\text { urinária e seu } \\
\text { impacto na } \\
\text { qualidade de vida } \\
\text { de mulheres } \\
\text { idosas residentes } \\
\text { na comunidade } \\
\text { que vivem em } \\
\text { populações } \\
\text { urbanas e rurais. }\end{array}$ & $\begin{array}{l}16 \text { a } 23 \% \text { entre } \\
\text { idosas rurais e } \\
\text { urbanas, } \\
\text { respectivamente. }\end{array}$ & $\begin{array}{l}\text { A IU é mais prevalente e } \\
\text { teve um impacto mais } \\
\text { profundo na qualidade da } \\
\text { saúde entre as mulheres } \\
\text { idosas no meio rural. Os } \\
\text { fatores de risco da IU foram } \\
\text { etnia e constipação crônica } \\
\text { entre mulheres idosas } \\
\text { urbanas e rurais, } \\
\text { respectivamente. }\end{array}$ \\
\hline $\begin{array}{l}\text { Mourão et } \\
\text { al. (2017) }\end{array}$ & $\begin{array}{l}\text { Caracterizar o } \\
\text { perfil } \\
\text { sociodemográfico, } \\
\text { epidemiológico e } \\
\text { clínico de } \\
\text { mulheres com IU } \\
\text { atendidas em uma } \\
\text { clínica no Piauí. }\end{array}$ & $41 \%$ em idosas & $\begin{array}{l}\text { Os principais fatores de } \\
\text { risco para IU apresentados } \\
\text { pelas participantes do } \\
\text { estudo foram: idade, } \\
\text { consumo de cafeína, prática } \\
\text { de atividade física, doenças } \\
\text { familiares, antecedentes } \\
\text { pessoais, ITU. }\end{array}$ \\
\hline
\end{tabular}




\begin{tabular}{|c|c|c|c|}
\hline $\begin{array}{l}\text { Santos, } \\
\text { Santo } \\
(2010)\end{array}$ & $\begin{array}{l}\text { Analisar fatores } \\
\text { Associados a IU } \\
\text { em idosos, com } \\
\text { critério de } \\
\text { fragilidade (frágeis } \\
\text { e pré-frágeis). }\end{array}$ & $65 \%$ & $\begin{array}{l}\text { Os dados apontaram como } \\
\text { fatores de risco a infecção } \\
\text { urinaria, perda de } \\
\text { mobilidade e sexo. }\end{array}$ \\
\hline $\begin{array}{l}\text { Silva et al. } \\
\text { (2012) }\end{array}$ & $\begin{array}{l}\text { Conhecer a } \\
\text { prevalência da } \\
\text { incontinência } \\
\text { urinária (IU), dos } \\
\text { fatores } \\
\text { demográficos e } \\
\text { clínicos preditores } \\
\text { da presença de IU. }\end{array}$ & $39,1 \%$ & $\begin{array}{l}\text { Permitiu concluir que as } \\
\text { prevalências padronizadas } \\
\text { de IU, segundo o sexo e } \\
\text { idade, em amostra de } 519 \\
\text { pessoas, foram } 20,1 \% \text { na } \\
\text { população geral, } 32,9 \% \\
\text { entre as mulheres e } 6,2 \% \\
\text { entre os homens. }\end{array}$ \\
\hline $\begin{array}{l}\text { Virtuoso et } \\
\text { al. (2015) }\end{array}$ & $\begin{array}{l}\text { Analisou fatores } \\
\text { de risco para } \\
\text { incontinência } \\
\text { urinária (IU) em } \\
\text { mulheres idosas } \\
\text { praticantes de } \\
\text { exercícios físicos } \\
\text { (EF). }\end{array}$ & $32,2 \%$ & $\begin{array}{l}\text { - Os achados apontaram o } \\
\text { uso de diuréticos e histórico } \\
\text { familiar positivo como } \\
\text { principais fatores de risco } \\
\text { para desenvolvimento de IU } \\
\text { em mulheres idosas } \\
\text { praticantes de EF. } \\
\text { - Sendo que ambos os } \\
\text { fatores não são os mesmos } \\
\text { comumente encontrados na } \\
\text { literatura. }\end{array}$ \\
\hline
\end{tabular}

Os estudos utilizaram medidas de avaliação variadas, através de questionários socioeconômicos, avaliação demográfica, nível de escolaridade, questionário sobre uso de medicamentos, história das doenças crônicas e também se utilizou análises de micção, presença de bacteriúria, escala de regressão de Poisson para análise bruta das idosas. 
Em alguns estudos foram feitas análises de prontuários, Teste do Qui-quadrado de Pearson avaliada por rastreamento de depressão a partir do The Center for Epidemiologic Studies - Depression (CES-D), adaptados no Brasil, que permite a identificação dos sintomas do entrevistado no período de três ou mais dias da semana anterior ao aparecimento de sintomas.

\section{DISCUSSÃO}

A busca pelo tema da IU em idosos do sexo feminino se deu devido a relevância do tema diante das dinâmicas sociodemográficas, pelas quais vive o Brasil atualmente. A transição demográfica brasileira, mostra que o envelhecimento populacional é um fato e junto a esse processo surge algumas síndromes geriátricas dentre elas está a incontinência urinaria (IU), cada dia mais presente na vida da população idosa. ${ }^{10} \mathrm{~A}$ origem da IU decorre de vários fatores, e de acordo com algumas pesquisas científicas a ocorrência pode acontecer devido às cirurgias ginecológicas, idade e analogia e também podem estar relacionadas à hipertensão arterial, diabetes mellitus, número parto normais e infecção do trato urinário. ${ }^{11}$

Autores ${ }^{6}$ observaram que o uso de medicamentos como diuréticos, vasodilatadores, anti-histamínicos, sedativos, tranquilizantes e narcóticos podem originar distúrbio de incontinência urinária. Porém os efeitos colaterais desses podem ser facilmente confundidos com sintomas de IU uma vez que fármacos como os diuréticos estimulam a diurese. ${ }^{12}$

Todavia, a incontinência urinária pode estar associada a diversas causas como: o enfraquecimento do músculo da bexiga, à diminuição da capacidade da bexiga que supostamente aumenta a frequência urinária, à redução de possibilidades de locomoção que dificultando as idas ao banheiro, ao enfraquecimento dos músculos pélvicos (na mulher) e aumento da próstata (no homem), à obstrução da uretra, doenças envolvendo nervos e/ou músculos, além disso, algumas cirurgias podem levar ao quadro de IU. ${ }^{12,13}$ 
Nos estudos levantados, foi possível observar que existe uma prevalência de mulheres idosas com incontinência urinária, ou seja, sabe-se que o número de idosos no Brasil vem crescendo e que o número de mulheres idosas no país é ainda maior, nesse sentido, tudo leva à evidência de que o número de mulheres idosas acometidas de IU é elevado, esses dados foram observados. ${ }^{14,5,9}$

Além disso, pesquisadores ${ }^{6}$ relatam que muitas dessas mulheres possuem problemas de saúde secundários e confluentes à IU, como: diabetes, hipertensão, usam medicamentos, números partos normais, infecção urinária, algumas possuem problemas com obesidade, e apresentam alterações hormonais que são fatores de risco para o surgimento de IU. Já questões socioeconômicas, condições de saúde e estilo de vida também são apontados como fator de risco para desenvolver a patologia, assim como, o consumo de cafeína, doenças familiares, antecedentes genéticos, infecções urinarias recorrentes e a obesidade podem estar associados com o surgimento de IU.7

Autores, ${ }^{5}$ apontam como fator de risco para IU, o comprimento da uretra feminina, a redução da pressão do fechamento uretral, o enfraquecimento do assoalho pélvico devido ao número de gestações e de parto e alterações hormonais que ocorrem com o avanço da idade. De acordo com pesquisadores ${ }^{1}$, os fatores predominantes para apresentar incontinência urinária está relacionado com a questão da idade e doenças como a diabetes e mal de Parkinson. No entanto, a obesidade é apontada como fator de risco, uma vez que o aumento do índice corporal no abdômen é um fator para ter incontinência urinária, consigna devido ao elevado acúmulo de gordura no abdômen. Já outros estudos, ${ }^{12}$ mencionam que os idosos são propensos a terem infecção da pele, disfunção sexual, perda de autoestima, dependência, depressão, fragilidade, institucionalização, aumento da carga do cuidador e custo econômico elevado. Nesse mesmo estudo sobre fatores associados a IU em idosos com critério de fragilidades, os autores citados também concluíram que a ITU é um fator que pode estar associado a IU em idosos frágeis e pré-frágeis, sendo que, as chances podem ser maiores quando esses idosos fazem uso de fraldas. ${ }^{4}$ 
Portanto, a Incontinência Urinária é uma situação clínica que tem recebido pouca importância tanto dos portadores da doença como dos responsáveis governamentais da saúde nacional. É importante perceber que essa patologia afeta de forma significativa a vida pessoal de cada paciente e interfere diretamente na socialização dessas pessoas, pois, além dos problemas físicos, esses indivíduos também apresentam o psicológico prejudicado no decorrer da vida. ${ }^{14}$ São diversos os fatores que podem levar à IU, dentre elas, as mais citadas pelas literaturas pesquisadas foram: idade, sexo, estilo de vida, obesidade, diabetes, pessoas praticantes de atividades físicas, número de infecções recorrentes do trato urinário, número de partos, antecedentes cirúrgicos, uso de diuréticos, consumo de cafeína, pessoas em situação de fragilidade que fazem uso ou não de fraldas, pessoas com déficit cognitivos e idosos em situação asilar. ${ }^{4,5,6,8,12,11}$ Logo, a incontinência urinária se desenvolve a partir de vários fatores. ${ }^{2,6,3,1,14}$ ou seja, é uma patologia multifatorial muito presente nos dias atuais.

Embora os instrumentos de avaliações utilizado pela maioria dos autores tenham sido diferentes, é possível observar que a prevalência da IU varia de 3 a $97 \%^{1,1} 14$ dependendo do local onde é feito a pesquisa, as instituições asilares possuem alta ocorrência e com maior índice no sexo feminino. ${ }^{4}$ Existem diversos artigos sobre a IU, porém poucos fazem relação com o surgimento de ITU, talvez pelo fato da maioria dos pacientes acometidos por essas patologias serem idosos frágeis em situação institucionalizada. Nesse caso, precisariam de mais pesquisas e estudos de caso, para aprimorar as teses em torno do tema.

\section{CONCLUSÃO}

No apanhado teórico acima, foi possível observar que a Incontinência Urinaria (IU) é uma patologia que está cada vez mais presente na vida das pessoas, na sua maioria, os acometidos são pessoas idosas e, principalmente, do sexo feminino. O fato do aparecimento e desenvolvimento da Incontinência Urinária ser confundida como um sinal de envelhecimento acaba fazendo com que essa doença seja passada despercebida pelos familiares e pessoas próximas que lidam diretamente com esses idosos. 
Além disso, o que dificulta ainda mais o diagnóstico é que muitas pessoas, incluindo idosos e idosas, sentem vergonha de relatar tal problema e acabam omitindo os sintomas, à vista disso, pode ocorrer um possível agravamento do quadro patológico.

Portanto, faz-se necessário que haja mais pesquisas sobre os casos de incontinência urinária, principalmente, em idosos, pois, há a necessidade de consolidar a prevenção e os tratamentos diante dos casos que já ocorrem no país e no mundo. É importante que essas pesquisas visem a prevenção e a orientação sobre os sinais e sintomas mais recorrentes, principalmente, para que ocorra a melhoria do diagnóstico e tratamento dessa patologia.

\section{REFERÊNCIAS}

1. CARVAlHO, P. M.; ANDRADE, P. F.; PERES, W.; MARTINELI, T.; SIMCH, ; ORCY, B. R.; SELEME, R. M. O impacto da incontinência urinária e seus fatores associados em idosas. Rev. Bras. Geriatr. Gerontol. Rio de Janeiro, v. 17, n. 4, 2014.

2. JUNQUEIRA, J.B.; SANTOS, V.L.C.G. Incontinência urinária em pacientes hospitalizados: prevalência e fatores associados. Rev. Lat. Am. Enfermagem, v.1, n.2, 2018.

3. CARNEIRO, J.A et al. Prevalência e fatores associados á incontinência urinária em idosos não institucionalizados. Cad. Sáude colet., v. 25, n. 3, 2017.

4. MELO, L.S; ERCOLE, F.F; OLIVEIRA, D.U; PINTO, T.S; VICTORIANO, M.A; ALCOFORADO, C.L.G.C.Infecção do trato urinário: uma coorte de idosos com incontinência urinária. Rev. Bras. Enferm., Brasília, v.70,n.4,2017.

5. SILVA, A. V.; D’ELBOUX, J. M. fatores associados á incontinência Urinaria em idosos com critérios de fragilidade. Rev. Texto e contexto-enfermagem. v. 21 no.2, 2012.

6. VIRTUOSO, F. J.; MENEZES, C. E.; MAZO, Z. G. Fatores de risco para incontinência urinária em Mulheres idosas praticantes de exercícios físicos, Rev. Bras. Ginecol. Obstet. v. 37 n.2, 2015. Acesso em 20 out. 2019.

7. MOURÃO, L.F; LUZ, M.H.B.A; MARQUES, A.D.B; BENICIO, C.D.A.V; BENEVINA, M.V.T.N; PEREIRA, A.F.M. Caracterização e fatores de risco de 
incontinência urinária em mulheres atendidas em uma clinica ginecológica. Rev. Estima, v.15, n. 2, 2017

8. SANTOS, C.R.S.; SANTOS, V. L.C.G. Prevalência da incontinência urinária em amostra randomizada da população urbana de Pouso Alegre, Minas Gerais, Brasil. Rev. Latino- Am. Enfermagem, v.18, n. 5, 2010.

9. BOLINA, A.F; DIAS, F.A; SANTOS, N.M.F; TAVARES, D.M.S. Incontinência urinaria auto-referida em idosos e seus fatores associados. Rev. Rene.Vol 14, ํำ, 2013.

10. MARQUES, P. L.; SCHNEIDER, C. J.; GIEHL, C. W. M.; ANTES, L. D.; D'ORSI, E. Fatores demográficos, de saúde e estilo de vida associados à incontinência urinária em idosos de Florianópolis. Rev.Bras. Epidemiologia. v. 18 no.3, 2015.

11. MENEZES, E. C; VIRTUOSO, J.F; CAPELETTO, E; SILVA, L.L; CHAGAS, J.M; MAZO. G.Z. Precisão diagnóstica de indicadores antropométricos na predição de incontinência urinária em mulheres idosas fisicamente ativas. Rev. Bras. Ginecol. Obstet. vol.38 no.8 Rio de Janeiro ago. 2016.

12. MURUKESU, R,R; SINGH, D.K.A; SHAHAR, S. Urinary incontinence amonyur banand rural community dwelling olderwomen: Prevalence, risk factor e sand quality of life. Rev. BMC public health. Vol.19 (supl. 4). 2019.

13. CAVALCANTE, K.V.M; SILVA, M.I.G.C; BERNADO, A.S.F; SOUZA, D.E; LIMA, T.C.G.C; MAGALHAES, A.G. Prevalência e fatores associados á incontinência urinaria em mulheres idosas. Rev. Bras. em promoção a saúde, vol. 27, n.2, 2014.

14. KESSLER, M.; FACCHINI, A. L.; SOARES, U. M.; NUNES, P. B.; FRANÇA, M. S.; THUMÉ, E. Prevalência de incontinência urinária em idosos e relação com indicadores de saúde física e mental. Rev. Bras. Geriatr. Gerontol. v. 21, n.4, 2018.

Enviado: Dezembro, 2020.

Aprovado: Julho, 2020. 